SIMPÓSIO: Tópicos fundamentais para a formação e o desenvolvimento docente para professores dos cursos da área da saúde Capítulo XI

\title{
Avaliação formativa e feedback como ferramenta de aprendizado na formação de profissionais da saúde
}

\section{Formative assessment and feedback as learning tools in health profes- sions education}

\author{
Marcos C. Borges ${ }^{1}$, Carlos H. Miranda ${ }^{2}$, Rodrigo C. Santana ${ }^{1}$, Valdes R. Bollela ${ }^{1}$
}

\begin{abstract}
RESUMO
As diferentes formas de avaliação são elementos centrais do processo de ensino-aprendizagem de qualquer programa educacional, e devem ser bem planejadas e implementadas em todas as propostas curriculares, especialmente na formação de profissionais na área da saúde. Uma avaliação do estudante adequada e de qualidade guarda estreita relação com a competência e capacitação do profissional que será entregue à sociedade. Neste contexto, a avaliação formativa e a capacitação dos professores para prover feedback efetivo, frequente, e de qualidade são fundamentais na formação dos futuros profissionais da saúde. Este artigo faz uma revisão sobre avaliação formativa, feedback e debriefing.
\end{abstract}

Palavras-chave: avaliação formativa, feedback, ensino médico.

\section{Principais pontos de interesse}

- A avaliação somativa e a formativa são formas complementares que visam conhecer e garantir os melhores resultados de processos e programas educacionais.

- A avaliação formativa estimula a autorregulação do estudante e, consequentemente, o desenvolvimento de habilidades para a educação permanente em saúde.

- O feedback é a atividade central da avaliação formativa.

- O feedback efetivo é uma das estratégias educacionais e avaliativas com maior evidência de eficácia na educação das profissões na área da saúde.

- Os professores e/ou preceptores de cursos na área da saúde precisam ser treinados para prover feedback efetivo.

- Estratégias de avaliação formativa devem ser incluídas no currículo de qualquer programa educacional para formar profisisonais na área da saúde.
1. Professor Doutor, 2. Médico Assistente. Departamento de Clínica Médica, Faculdade de Medicina de Ribeirão Preto da Universidade de São Paulo
Correspondência: Prof Dr. Valdes Bollela Departamento de Clínica Médica, FMRP-USP Av. Bandeirantes, 3900 CEP: 14049-900 - Ribeirão Preto, SP

Artigo recebido em 22/05/2014 Aprovado para publicação em 19/06/2014 


\section{Introdução}

$\mathrm{O}$ ato de avaliar encerra uma multiplicidade de significados e interpretações: examinar, julgar, testar, distinguir, comparar, ameaçar, punir, entre outros. E é por esta variedade de abordagens que o processo de avaliar se torna tão difícil, ficando a mercê do julgamento de quem a pratica. Entretanto, o tema assume hoje papel de destaque nas discussões pedagógicas em diferentes cenários de ensino, onde se busca metodologias mais eficientes e melhor padronização das diferentes formas de avaliação.

O processo de avaliação se desenvolve paralelamente à própria história da humanidade. Podemos citar maneiras tribais primitivas de se avaliar (selecionar), como por exemplo, o momento mais apropriado para um jovem se tornar adulto ou para que determinado indivíduo assumisse um cargo de prestígio nas sociedades antigas ${ }^{1}$, até estratégias de avaliação, no contexto educacional, que evoluíram e possuem uma compreensão global, menos pontual e mais voltada ao processo de avaliar estudantes nos cenários de ensino-aprendizagem. Esta compreensão vem sendo debatida exaustivamente e tem sido gradualmente assimilada nos círculos pedagógicos do ensino básico. ${ }^{2}$ No entanto, no ensino superior, a evolução dos métodos de avaliação ainda encontra obstáculos e demora para se instalar plenamente. Dentre os fatores que contribuem para esta resistência estão limitações na formação didática dos professores, rigidez na estrutura curricular, sobrecarga de funções e, consequentemente, falta de motivação pelo corpo docente em debater novas metodologias de ensino-aprendizagem e avaliação.

Essas dificuldades assumem particular relevância nos cursos de graduação em medicina e nas outras profissões da saúde, onde os diferentes cenários de ensino-aprendizagem, as inúmeras atribuições dos docentes e a complexidade da estrutura curricular tornam o desafio de mudanças ainda maior. Neste contexto, os métodos de avaliação formativa se projetam como estratégias mais abrangentes e menos pontuais que os tradicionais métodos somativos de avaliação, contribuindo para a formação de profissionais mais autônomos e reflexivos.

\section{Díferenciação de avaliação somativa e formativa}

Tradicionalmente, a avaliação do estudante nos cursos de graduação na área da saúde tem sido reali- zada de maneira pontual, geralmente ao final de um módulo ou curso. Essa modalidade de avaliação, denominada somativa, tem como objetivo avaliar se o aluno assimilou os conteúdos fornecidos durante determinado período. Também apresenta caráter classificatório e certificativo, ou seja, o aluno deverá atingir determinada pontuação para ser aprovado e, inevitavelmente, é comparado aos seus pares. Esse sistema de avaliação pressupõe e reforça o conceito de que o bom aluno é aquele que atinge elevada pontuação nos testes. Além disso, ele considera que, ao se iniciar o curso, todos estão em igual condição de aprender os conteúdos ensinados.

Inúmeras são as críticas a este formato de avaliação quando usado exclusivamente, entre elas, que a avaliação somativa foca mais no resultado final do que na trajetória percorrida pelo estudante durante a aquisição dos conhecimentos e habilidades. Apesar do caminho a ser percorrido pelos estudantes ser o mesmo, a velocidade e a experiência de cada um fazem com que a trajetória percorrida não seja a mesma para todos. Nesse sentido, a formação de um estudante deveria ser entendida como um processo que precisa ser avaliado continuamente. Desta forma, com este tipo de avaliação não seria possível detectar e oferecer, em tempo adequado, soluções para corrigir eventuais dificuldades enfrentadas pelo estudante. Considera-se ainda, que o caráter classificatório da avaliação somativa a torna julgadora e até discriminatória, ao determinar que aqueles com alguma dificuldade na aprendizagem seriam os "maus alunos".

Apesar das limitações descritas acima, é importante ressaltar que a avaliação somativa apresenta características interessantes e tem um importante papel no currículo dos cursos na área da saúde, pois, quando bem aplicada, tem uma excelente capacidade para avaliar aquisição de conhecimentos e habilidades, sendo uma das estratégias mais apropriadas e utilizadas para decidir sobre a progressão e/ou certificação dos alunos.

Por outro lado, a avaliação formativa pressupõe que o ato de avaliar não faz sentido por si só, e sim que ele deve ser parte integrante de todo processo de ensino-aprendizagem. Para tanto, a avaliação deve ser contínua e não mais pontual. Nesse sentido, todas as informações produzidas pela interação de professores e alunos, bem como entre os alunos, são relevantes para a verificação do grau de aprendizado e para eventuais ajustes necessários a fim de que o estudante consiga atingir os objetivos definidos (ver figura 1). Com isso, a avaliação passa a ser uma ativi- 


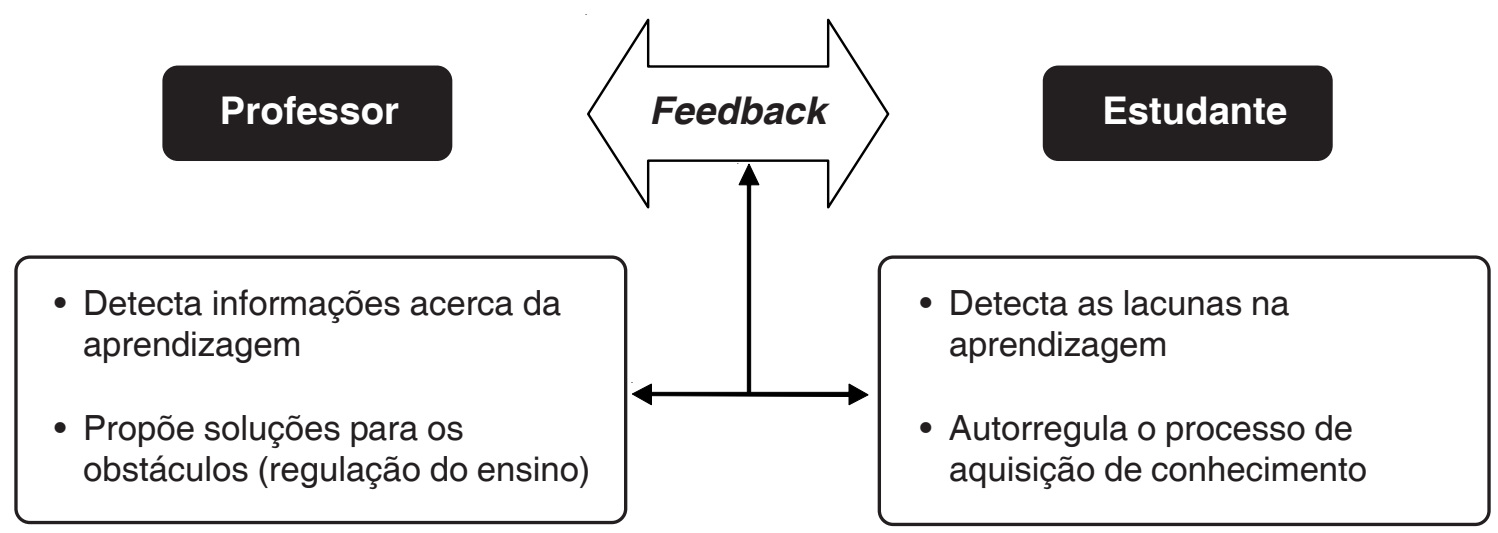

\section{Ensino-aprendizagem}

Figura1. Papel do professor e estudante no processo de ensino-aprendizagem segundo o modelo de avaliação formativa.

dade reguladora do processo de ensino-aprendizagem, detectando lacunas e proporcionando soluções para eventuais obstáculos enfrentados pelos estudantes, além de proporcionar melhorias nas ferramentas didáticas e eventuais ajustes no conteúdo programático ou mesmo na estrutura curricular. ${ }^{3}$

Um dos componentes principais da avaliação formativa é o feedback. ${ }^{4} \mathrm{O}$ feedback regula o processo de ensino-aprendizagem, fornecendo, continuamente, informações para que o estudante perceba o quão distante, ou próximo, ele esta' dos objetivos almejados. $\mathrm{O}$ fato de o feedback ser contínuo permite que os ajustes necessários para a melhor qualidade da aprendizagem sejam feitos precocemente, e não apenas quando o aluno falha no teste ao final do curso, ou seja, na avaliação somativa. Se por um lado ofeedback é essencial, ele não garante a aprendizagem sem que haja adequado estímulo aos processos cognitivos e metacognitivos do estudante, que devem ser o centro do processo de ensino-aprendizagem. ${ }^{5}$ Isso significa que o estudante deve ser estimulado a desenvolver a autoavaliação e a autorregulação do seu aprendizado. Assim o aluno se compromete com o esforço necessário para reduzir a distância entre o que ele sabe e o que é capaz de fazer, entre o nível de aprendizado que está e o nível de capacitação a ser atingido. É neste ponto que ofeedback do professor pode produzir o maior benefício. O feedback também deverá estimular o desenvolvimento da capacidade reflexiva e autoavaliativa dos estudantes. ${ }^{3}$ Isto ocorre pois, ao receber ofeedback o estudante deve refletir sobre seu próprio desempenho e como incorporar as novas práticas sugeridas para melhorá-lo no futuro.

Assim, o conceito mais aceito atualmente sobre boas práticas em termos de avaliação do estudante/residente é o da complementaridade entre as duas formas: somativa e formativa. A Tabela 1 mostra as principais características de cada uma delas e como se complementam.

Por fim, conclui-se que a avaliação formativa, escopo desse artigo, é uma das mais importantes ferramentas pedagógicas existentes e que, se bem utilizada, se transforma num dos componentes centrais do processo de ensino-aprendizagem. Entretanto, a implementação de métodos apropriados de avaliação formativa ainda encontra diversos obstáculos para ser regularmente utilizada nos cursos de graduação na área da saúde. Dentre estes, estão a falta de conhecimento a respeito das metodologias de avaliação, limitações na formação didática do professor, maior valorização das atividades de pesquisa em detrimento das acadêmicas, rigidez da estrutura curricular, e mesmo a falta de um referencial conceitual claro e preciso acerca das práticas de avaliação formativa nos cursos de graduação na área da saúde. ${ }^{6}$

\section{Feedback como atividade estrutu- rante da avaliação formativa}

Aprendemos no estudo da fisiologia humana sobre a existência de importantes mecanismos de autorregulação (feedback) que garantem o perfeito 
Tabela 1: Comparação entre as características básicas das avaliações somativa e formativa.

\begin{tabular}{|c|c|}
\hline AVALIAÇÃO SOMATIVA & AVALIAÇÃO FORMATIVA \\
\hline $\begin{array}{l}\text { PONTUAL } \\
\text { - Geralmente aplicada ao final de um curso ou em } \\
\text { momentos definidos. }\end{array}$ & $\begin{array}{l}\text { CONTÍNUA } \\
\text { - Realizada durante os momentos de interação en- } \\
\text { tre os professores e os alunos. }\end{array}$ \\
\hline $\begin{array}{l}\text { FORMAL } \\
\text { - Realizada em um momento definido, normalmen- } \\
\text { te o dia definido para a realização da prova. }\end{array}$ & $\begin{array}{l}\text { INFORMAL } \\
\text { - Realizada naturalmente durante todos as oportu- } \\
\text { nidades de interação entre professores e alunos, e } \\
\text { em diferentes cenários. }\end{array}$ \\
\hline $\begin{array}{l}\text { ESTÁTICA } \\
\text { - Pré-estabelecida no início do curso. Avaliará se o } \\
\text { estudante adquiriu os conhecimentos e habilida- } \\
\text { des esperados. }\end{array}$ & $\begin{array}{l}\text { DINÂMICA } \\
\text { - Permite ajustes durante o curso, corrigindo os } \\
\text { eventuais obstáculos enfrentados pelos alunos na } \\
\text { aquisição dos objetivos. }\end{array}$ \\
\hline $\begin{array}{l}\text { JULGADORA OU HIERARQUIZADORA } \\
\text { • Define com base nas pontuações quem são os } \\
\text { "bons" e os "maus" alunos. Favorece a competi- } \\
\text { ção entre os estudantes. }\end{array}$ & $\begin{array}{l}\text { NÃO JULGADORA } \\
\text { - Considera a individualização no processo de } \\
\text { aprendizagem. Favorece a auto-estima entre os } \\
\text { estudantes. }\end{array}$ \\
\hline $\begin{array}{l}\text { TOMAR DECISÃO } \\
\text { - Utilizada para decidir sobre a progressão e/ou } \\
\text { certificação. }\end{array}$ & $\begin{array}{l}\text { AUXILIAR NO APRENDIZADO } \\
\text { - Pela própria natureza da avaliação é parte da es- } \\
\text { tratégia de ensino-aprendizagem }\end{array}$ \\
\hline
\end{tabular}

funcionamento de complexos sistemas biológicos com o objetivo de manter a homeostase do indivíduo. Esta é a essência do conceito de feedback no contexto da educação. O feedback deve ser encarado como um processo onde tanto o professor quanto o aluno se modificam nas atividades de ensinar e aprender, o que permite a criação de um ambiente propício a discussão de ideias e ao aprimoramento de habilidades. De maneira mais objetiva, o feedback referese à informação que será dada ao aluno para descrever e avaliar o seu desempenho em uma determinada atividade, comparando o resultado observado com aquele que realmente era esperado que ele obtivesse, que deve ser baseado em premissas pré-estabelecidas de competências para aquele determinado grau de formação.

Por exemplo, durante a formação médica nos deparamos com alunos brilhantes, estudiosos, que conseguiram excelentes notas nos exames de admissão, mas que durante o andamento do curso vão perdendo o interesse por novos conhecimentos, ficam desmotivados e não conseguem colocar em prática o que aprenderam. Este comportamento pode ser refle- xo de um ambiente de ensino desestimulante, onde o foco está apenas no acúmulo de informações. Neste contexto, a atividade de feedback surge como uma importante ferramenta de orientação para uma formação mais ampla com intuito de qualificar o aprendizado e aplicar o conhecimento adquirido, rumo a competência profissional.

Em nossa experiência, acreditamos que o feedback, tal como deve ser feito, é realizado em raras oportunidades. E quando é feito, geralmente somente os pontos negativos da atuação do aluno são destacados, criando um ambiente hostil onde é enfatizada a superioridade do professor e não se abre espaço para o diálogo, ou seja, somente funciona a alça inibitória do professor sobre o aluno. O conceito de feedback exige uma interação aberta entre o professor e o aluno, e tem como ponto fundamental o diálogo, que deve ocorrer de maneira informal, dinâmica, desprovido de preconceitos e sempre presente no processo de ensino-aprendizagem.

Em relação à frequência, as boas práticas em termos de avaliação formativa, recomendam que o feedback seja oferecido regularmente, de modo a ofe- 
recer oportunidades para o estudante refletir e rever suas práticas ainda durante a experiência educacional. Além de frequente, o feedback deve ter qualidade. Um feedback mal realizado poderá causar prejuízos à formação do aluno, além de estimular o comportamento defensivo e o desinteresse. Para prover feedback adequado o preceptor deve ter conhecimento, atitude e postura adequadas para ensinar, empatia com o aluno, e habilidade para avaliar naquele cenário de aprendizagem.

\section{Como prover um feedback efetivo?}

Antes de mais nada é preciso estabelecer, desde o início, quais são objetivos de aprendizagem e competências a serem adquiridos em um determinado cenário de ensino. Assim, este referencial deve nortear todas as práticas de ensino e a própria avaliação e feedback.

Sempre que possivel, o estudante deve receber feedback sobre seu desempenho. Neste sentido, nos cursos na área da saúde, os estágios que se passam nos cenários reais da prática (ex. unidades de saúde, ambulatórios e enfermarias) são os principais momentos em que o aprendiz pode qualificar e melhorar sua prática, a partir da devolutiva (feedback) dos preceptores que o acompanham e supervisionam.

As características desejáveis do feedback na prática são:

- Oportuno: o feedback deverá ocorrer o mais próximo possível do evento ou da atividade programada, pois quanto mais tempo se passa, detalhes importantes da observação do aluno ou do professor podem ser perdidos.

- Restrinja-se ao que foi observado: comente somente pontos observados naquele encontro, evitando julgamentos e comentários dirigidos à personalidade do estudante ou baseados em opiniões préconcebidas, oriundos de outras situações vivenciadas previamente com o aluno.

- Comece solicitando uma autoavaliação: deixe sempre o aluno falar primeiro, esta é uma excelente oportunidade para avaliar a capacidade de autorreflexão e ver se o estudante consegue identificar seus pontos fracos.

- Seja específico: tente ser o mais específico e descritivo possível em seus comentários. Evite frases prontas e sem conteúdo formativo, como "Você foi muito bom", "Bom trabalho”. Prefira comentários como, por exemplo: "A forma com que você conduziu a anamnese foi apropriada para esta situação devido a dificuldade de comunicação com este paciente na fase aguda do acidente vascular cerebral".

- Comece pelos pontos positivos observados: nunca comece o feedback ressaltando os pontos negativos, isto pode levar a um bloqueio no fluxo de ideias por parte do aluno e gerar um ambiente defensivo. Ressalte, inicialmente, os pontos fortes, pois isto abre um canal de comunicação e empatia. Uma estratégia que pode ser utilizada é fazer um comentário negativo entre duas observações positivas, esta abordagem é conhecida com "técnica do sanduíche" e pode ser particularmente útil para alunos mais resistentes.

- Evite dar um grande volume de feedback negativo de uma só vez: quando existem vários pontos negativos, tente focar em um ponto mais importante ou central e que seja mais fácil de ser corrigido e remediado durante aquela sessão.

- Crie um ambiente acolhedor: tente criar um ambiente estimulante e acolhedor, onde as pessoas tenham liberdade de manifestar suas dúvidas e fraquezas, para isto, evite tentar convencer com seus argumentos, evite falta de clareza e de honestidade em suas considerações.

Os comentários devem surgir como recomendações de uma pessoa mais experiente que já vivenciou uma situação semelhante e não como uma crítica a atuação do aluno. Por exemplo, evite dizer:

"Você deve ser mais objetivo nas questões for-

muladas para o paciente"

O mesmo comentário pode ser dito da seguinte forma:

"Durante a entrevista com o paciente nós devemos estimar o grau de instrução do paciente, como por exemplo, a linguagem do mesmo, e a partir de então nossas questões devem ser construídas da maneira mais clara possivel para o entendimento daquele paciente".

As recomendações mais utilizadas para prover um feedback de qualidade foram descritas por Pendleton em 1984. ${ }^{7}$ Elas são conhecidas como regras de Pendlenton e os seus principais pontos estão resumidos abaixo:

- Seja claro ao fazer qualquer pergunta ou comentário; 
- Peça ao aluno para comentar o que ele fez bem durante a avaliação ou discussão e porque ele chegou a esta conclusão;

- Se o feedback for fornecido em uma atividade em grupo, peça aos integrantes do grupo comentarem o que eles observaram o aluno fazer corretamente;

- Peça ao aluno para comentar o que ele não fez tão bem e como poderia ter feito diferente;

- Discuta o que poderia ter sido feito diferente e qual a melhor maneira de executar aquela tarefa.

O objetivo primordial do feedback é fornecer ferramentas para melhorar o desempenho do aluno, identificando seus pontos fracos e ajudando-o a criar alternativas para superá-los. Para ter qualidade, o feedback não precisa ser longo, mas precisa ser claro e objetivo e transferido da maneira mais adequada possível, despertando a reflexão do aluno, pois somente desta maneira conseguimos modificar algum comportamento. A Tabela 2 compara as características de uma feedback efetivo com as de um feedback inapropriado.

\section{Evidências de que o feedback funciona}

Existem várias evidências de que o feedback é efetivo em melhorar o aprendizado do aluno, resultando em melhor desenvolvimento profissional. Um estudo observou que alunos preferem ser avaliados por testes frequentes e que ofeedback proporcionado por estes testes repetidos melhoram o aprendizado. ${ }^{8,9}$ Algumas observações podem ser diretamente percebidas com o emprego desta técnica, como maior participação dos alunos nas atividades; melhoria nas habilidades de comunicação e de trabalho em grupo; e maior motivação em busca do conhecimento.

A falta de feedback distancia o aluno dos objetivos primordiais de sua formação, levando-o muitas vezes para uma interpretação errada de seu comportamento, o que pode gerar duas consequências extremas: desenvolvimento de uma "falsa confiança" ou insegurança na tomada de decisões. Estudos mostram que a utilização do feedback pode melhorar o desempenho do aluno na execução do exame físico e nas habilidades clínicas de uma forma geral. ${ }^{10,11,12}$ Especialistas em ensino enfatizam os benefícios do feedback no aprendizado, contudo o real impacto desta metodologia é de difícil mensuração, principalmente devido inúmeras variações que podem ocorrer dentro do contexto onde ele é aplicado.

Os alunos também reconhecem a importância do feedback em sua formação, como demostrado em um estudo que avaliou mais de 3 mil alunos no último ano do curso de medicina e residentes. Destes, 95\% consideraram dar e receber um feedback adequado como a segunda mais importante habilidade de ensino, sendo ultrapassada somente pela capacidade de estar aberto para perguntas. ${ }^{13}$

Tabela 2. Comparação das características de um feedback efetivo e um feedback inadequado.

\begin{tabular}{ll}
\hline FEEDBACK EFETIVO & FEEDBACK INADEQUADO \\
\hline Regular & Ocasional \\
Bidirecional & Unidirecional \\
Enfatiza pontos positivos e negativos & Enfatiza somente pontos negativos \\
Desperta autorreflexão & Não gera autorreflexão \\
Auxilia a melhorar o desempenho & Críticas em relação ao desempenho \\
Motivação para aprender & Ausência de motivação \\
É parte do processo de aprendizagem & Não agrega valor no aprendizado \\
Faz conexão entre o aprendizado e a realidade & Desconectado da realidade/prática diária \\
Aperfeiçoa as habilidades de ensinar e aprender & Mantém preconceitos \\
Foca no comportamento observado & Foca na personalidade \\
Observação e comentários específicos & Observação e comentários não específicos \\
\hline
\end{tabular}




\section{Debriefing}

O debriefing consiste em uma modalidade particular de feedback, sendo aplicado predominantemente em atividades de aprendizagem experiencial, como na simulação de tarefas. O termo debriefing originase da estratégia militar de obter informações de um piloto ou soldado, após retornar de uma missão, com o objetivo de usar os relatos para a geração de experiência militar e para facilitar a reintegração psicossocial dos soldados após a experiência de guerra. ${ }^{14}$ No contexto educacional, o debriefing se fundamenta no aprendizado gerado pela reflexão, individual e/ou em grupo, do desempenho após a realização de uma determinada tarefa. Dessa forma, o termo debriefing poderia ser melhor compreendido como "reflexão pósexperiência".

No ensino médico, a utilização da simulação na educação, bastante empregada em universidades norte-americanas e europeias ${ }^{15,16,17}$, tem ganhado relevância crescente no Brasil. ${ }^{18}$ Dentre os métodos simulados empregados no ensino em saúde, podemos citar a realização de procedimentos em manequins, como intubação orotraqueal, manobras de ressuscitação cardiorrespiratória, punções venosas, dentre outras. Assim, após a realização da tarefa segue-se a reflexão sobre o desempenho dos executantes. $\mathrm{O}$ resultado dessa reflexão servirá para melhorar o desempenho individual e/ou da equipe, configurando-se em uma valiosa ferramenta de aprendizagem. Além destas situações, o debriefing tem sido empregado também em situações mais complexas, como treinamento em situações críticas, especialmente em situações de emergência ou simulações de trabalho em equipe. ${ }^{18}$
Os componentes estruturais do debriefing são:

- Facilitador: responsável pela simulação, e mais importante, pela moderação da atividade de reflexão;

- Participantes: geralmente alunos, médicos e enfermeiros;

- Experiência gerada na equipe: relacionada ao cenário da simulação;

- Impacto gerado na equipe pela simulação: quanto mais relevante a simulação e quanto mais envolvidos por ela, melhor será o resultado no aprendizado;

- Recordação sumária dos principais eventos e ações durante a simulação;

- Relato verbal ou por escrito sobre o evento, com o intuito de mensurar o desempenho;

- Tempo decorrido entre a simulação e o debriefing: geralmente é realizado logo em seguida;

- Duração da sessão. ${ }^{18,19,20}$

A sessão de debriefing deve levar em conta os objetivos a serem aprendidos e as características dos participantes. Os objetivos devem estar bem definidos para que se identifique as eventuais lacunas no desempenho dos executantes. Neste contexto, o facilitador (debriefer) assume papel essencial. Ele deve ter habilidade suficiente para promover atitudes avaliativas e reflexivas dentre os participantes. Para isso, o facilitador deve assumir menos a postura de professor e de transmissor de conhecimento, e mais a de um facilitador do trabalho da equipe, rumo aos resultados esperados. Por sua vez, os participantes devem assumir uma postura menos passiva, de expectadores, e mais participativa, estando ativamente envolvidos na avaliação crítica do próprio aprendizado. ${ }^{19}$

\section{ABSTRACT}

The different assessment forms are major elements of any teaching and learning process in educational programs, and should be considered as a core component to be planned and implemented in all curriculums, especially in the health professions education. A regular and qualified students' assessment is closely related to competence and skills of the professionals that will be delivered to society. In this context, formative assessment and well-trained staff to provide effective and regular feedback are essentials in the formation of the future generation of health professionals. This article focuses primarily on formative assessment, feedback and debriefing.

Key-words: formative assessment, feedback, debriefing, medical education 


\section{Referências Bibliográficas}

1. Soeiro LReA, S. Avaliação educacional. Porto Alegre: Sulina, 1982.

2. Stainle MCeS, N.A. Avaliação formativa e o processo de ensino/aprendizagem na educação infantil. Est Aval Educ. 2007; 18:63-74.

3. Wood DFFA, in Understanding Medical Education: Evidence, Theory and Practice (ed T. Swanwick), Wiley-Blackwell, Oxford, UK. doi: 10.1002/9781444320282.ch18.

4. Rushton A. Formative assessment: a key to deep learning? Med Teach. 2005; 27:509-13.

5. Fernandes D. Para uma teoria da avaliação formativa. Rev Port Educ. 2006 19:21-50.

6. Norcini J, Anderson B, Bollela V, Burch V, Costa MJ, Duvivier $\mathrm{R}$, et al. Criteria for good assessment: consensus statement and recommendations from the Ottawa 2010 Conference. Med Teach. 2011;33:206-14.

7. Pendleton D, Schofield T, Tate P, Havelock P. The consultation: an approach to learning and teaching: Oxford University Press Oxford; 1984.

8. Iverson AM, Iverson GL, Lukin LE. Frequent, ungraded testing as an instructional strategy. J Exp Educ. 1994;62:93-101.

9. Scheerens J. Process indicators of school functioning: a selection based on the research literature on school effectiveness. Stud Educ Eval. 1991;17:371-403.

10. Newble DI, Jaeger K. The effect of assessments and examinations on the learning of medical students. Med Educ. 1983; $17: 165-71$

11. Papa FJ, Aldrich D, Schumacker RE. The effects of immediate online feedback upon diagnostic performance. Acad Med. 1999;74(10 Suppl):S16-8.
12. Zeferino AMB, Domingues RCL, Amaral E. Feedback como estratégia de aprendizado no ensino médico. Rev Bras Educ Méd. 2007;31:176-9.

13. Schultz KW, Kirby J, Delva D, Godwin M, Verma S, Birtwhistle $\mathrm{R}$, et al. Medical Students' and Residents' preferred site characteristics and preceptor behaviours for learning in the ambulatory setting: a cross-sectional survey. BMC Med Educ. 2004;4:12.

14. Pearson M, Smith D. Debriefing in experience-based learning. Reflection: Turning experience into learning. 1985:6984.

15. Awtrey CS, Fobert DV, Jones DB. The Simulation and Skills Center at Beth Israel Deaconess Medical Center. J Surg Educ. 2010;67:255-7.

16. Fraser K, Wright B, Girard L, Tworek J, Paget M, Welikovich $\mathrm{L}$, et al. Simulation training improves diagnostic performance on a real patient with similar clinical findings. Chest. 2011;139:376-81.

17. Stefanidis D, Acker CE, Greene FL. Performance goals on simulators boost resident motivation and skills laboratory attendance. J Surg Educ. 2010;67:66-70.

18. Flato UAP, Guimarães HP. Educação baseada em simulação em medicina de urgência e emergência: a arte imita a vida. Rev Bras Clin Med São Paulo. 2011;9:360-4.

19. Fanning RM, Gaba DM. The role of debriefing in simulationbased learning. Simulation in healthcare: journal of the Society for Simulation in Healthcare. 2007;2:115-25.

20. Lederman LC. Debriefing: Toward a systematic assessment of theory and practice. Simul Gaming. 1992;23:145-60. 